\title{
Research on Driving Control and Parameter Design of Hydrostatic Transmission Vehicle
}

\author{
Liang Zhao ${ }^{1}$, Liang Zhao ${ }^{2}$, Jin Wang ${ }^{2}$, Wei Ning ${ }^{2}$ \\ ${ }^{1}$ School of Construction Machinery, Chang'an University, Xi'an, China \\ ${ }^{2}$ School of Machinery Engineering, Shaanxi University of Technology, Hanzhong, China \\ E-mail: zhaoliangslg@163.com, wangjin63@163.com
}

Keywords: hydrostatic, hydraulic motor, hydraulic pump, parameter design

\begin{abstract}
In order to make the parameters of multi-axis hydrostatic transmission vehicle design more reasonable, the driving principle of hydrostatic transmission vehicle is introduced. According to the number of driving axles of hydrostatic transmission vehicle, the division of stall and parameter design are carried out. Control method of hydraulic motor. According to the driving equation of the vehicle, the driving force required by the system is determined, and the parameters of the hydraulic motor and the hydraulic pump are designed and calculated, which provides a reliable basis for the parameter design of the hydrostatic transmission vehicle.
\end{abstract}

\section{Introduction}

It is a special chassis for operation, such as concrete mixer truck chassis, concrete pump truck chassis and large fire engines, etc., must be a second type of chassis to be modified to meet the vehicle after the conversion requirements, such as full power Output force device, and even install the transfer case and vice engine. Hydraulic transmission technology with a wide range of speed, easy to control, can achieve stepless speed and other characteristics, can achieve the vehicle's stepless speed regulation and freestyle, in the construction machinery walking system has been a lot of applications. As the need for special chassis models are growing, engineering machinery and often in a certain work area for micro-operation. According to the characteristics of hydraulic transmission, the use of hydrostatic transmission of the way, so that the power transmission layout flexible and easy to achieve the vehicle's stepless speed.

\section{Composition and working principle}

The hydrodynamic drive vehicle has four hydraulic pumps and 14 drive motors, using a fully driven approach. The working principle of the hydraulic drive system is shown in Figure 1, which includes the engine 1 , the hydraulic pump 2, the fuel tank 3 , the cartridge valve 4 , the electromagnetic directional valve 5 , the relief valve 6 , the hydraulic motor 7 , Wheel reducer 8 and tire 9.

The engine 1 is connected to the hydraulic pump 2 to drive the hydraulic pump to rotate, to supply the hydraulic oil to the travel drive system, to drive the hydraulic motor 7 via the cartridge valve 4 , to rotate the wheel 9 by the wheel speed reducer 8 , The solenoid valve 5 controls the cartridge valve 4 to change the flow of the hydraulic oil so that the vehicle is advanced or retreated. The constant power control of the hydraulic pump with the pressure cut-off is used, and the displacement of the hydraulic pump varies depending on the rotational speed of the engine 1 . The hydraulic motor 7 is controlled by the electrical ratio, and the displacement of the hydraulic motor is controlled by the magnitude of the current of the given hydraulic motor coil. 


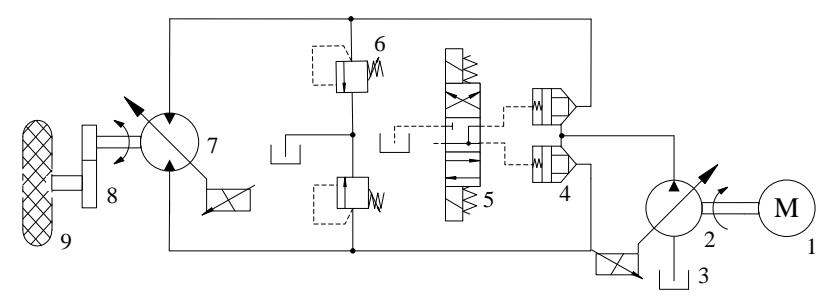

Figure 1. Schematic diagram of single-sided driving hydraulic system

\section{Driving principle}

The control of the drive drive section includes the control of the engine, the variable hydraulic pump, the variable hydraulic motor and the associated solenoid valve. The driving schematic diagram shown in Figure 2. The engine speed is adjusted by adjusting the throttle opening of the engine to adjust the throttle opening of the engine. The maximum power of the engine at each speed is determined according to the external characteristic curve of the engine, and the target displacement of the variable hydraulic pump is determined according to the pressure of the hydraulic system. Through the stall handle stall signal and hydraulic system pressure adjustment variable hydraulic motor displacement, change the speed of the variable hydraulic motor to change the speed of the vehicle.

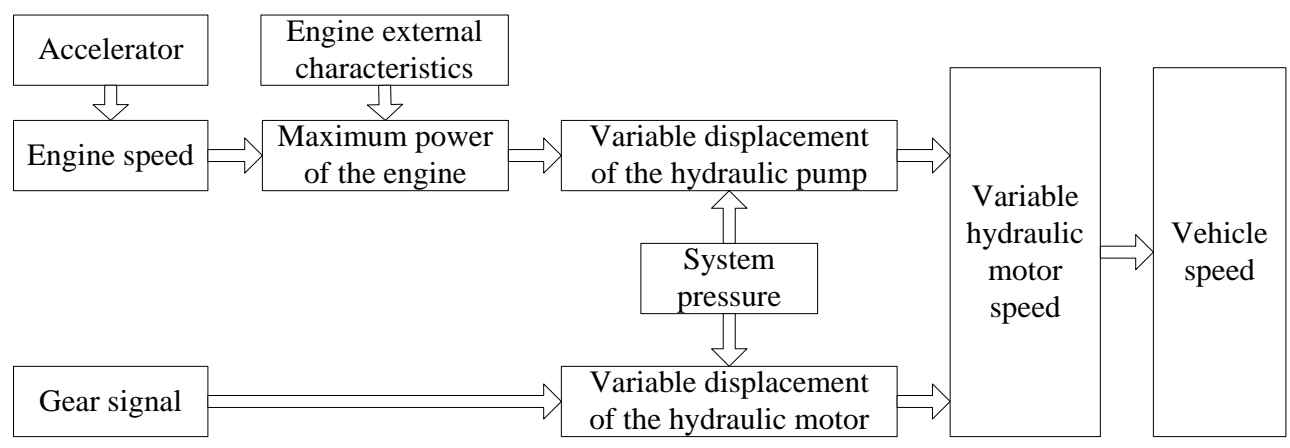

Figure 2. Travel control schematic

\section{Hydraulic pump and motor control}

\subsection{Stall division and parameter setting}

Table 1 a Gear and parameter list

\begin{tabular}{|l|c|c|c|c|c|}
\hline \multirow{2}{*}{\multicolumn{1}{|c|}{ Parameter }} & \multicolumn{5}{c|}{ Gear } \\
\cline { 2 - 6 } & 1st & 2nd & 3rd & 4th & 5th \\
\hline Speed $(\mathrm{km} / \mathrm{h})$ & $<22$ & $<30$ & $<43$ & $<56$ & $56 \sim 80$ \\
\hline Engine speed (rpm) & $<1400$ & $<1500$ & $<1700$ & $<1800$ & $<1900$ \\
\hline Pump displacement $(\mathrm{mL})$ & $<190$ & 190 & 190 & 190 & 190 \\
\hline Motor displacement (mL) & $200 \sim 53$ & $76-52$ & $65-52$ & $70-52$ & $78-52$ \\
\hline
\end{tabular}

There is no gearbox in the drive system, and the design of the gear is based on the number of drive axles. When the vehicle is moving forward, the number of axles is changed from 7 to 3 , and the number of axles is divided into five gears according to the number of drive axles. Can be manual and automatic gear switch, when the need to drive the number of less when the corresponding turn off the displacement of the hydraulic motor, let it in a zero displacement state, to improve the speed of other drive hydraulic motor. Parameters and gears are shown in Table 1. 


\subsection{Hydraulic pump control}

The displacement of the hydraulic pump for a single parameter control, according to the engine changes set the pump displacement, pump displacement and the engine speed $n$.

$$
q=f(n)
$$

Where $q_{b}$ is the displacement of the hydraulic pump; $n$ is the speed of the engine.

The relationship is determined by the following figure:

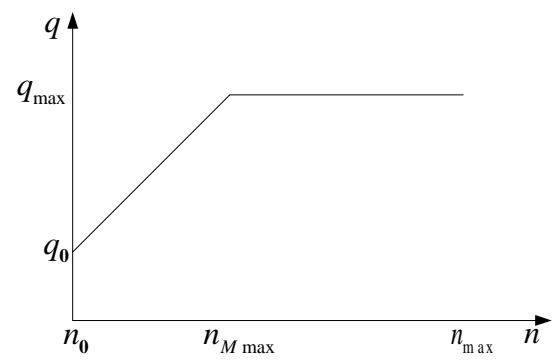

Figure 3. relationship between the displacement of pump and engine speed

Where $q_{0}$ is the minimum displacement of the hydraulic pump, $q_{\max }$ is the maximum displacement of the hydraulic pump, $n_{0}$ is the engine idling, $n_{M \max }$ is the maximum torque speed of the engine, $n_{\max }$ is the maximum engine speed.

\subsection{Hydraulic motor control}

Hydraulic motor displacement according to the stall, speed and system pressure to calculate the motor displacement, at any time, speed and force into a similar hyperbolic relationship, according to the current speed calculated motor target displacement:

$$
q_{m}=\frac{q_{\min } \cdot v_{\max }}{v}
$$

Where $q_{m}$ is the displacement of the hydraulic motor; $v$ is the speed of the vehicle; $v_{\max }$ is the maximum speed per file; $q_{\min }$ is the minimum displacement corresponding to each gear of the motor.

\section{Calculation of vehicle driving force}

Driving process, the chassis to provide the driving force and the resistance should be maintained in balance, the vehicle's driving equation is:

$$
F_{t}=F_{f}+F_{w}+F_{i}+F_{j}
$$

Where $F_{t}$ is the driving force; $F_{f}$ is the rolling resistance, $F_{f}=M g f ; F_{w}$ is the wind resistance, $F_{w}=\frac{C_{D} A v}{21.15}, C_{D}$ is the wind resistance coefficient, $A$ is the windward area of the vehicle, $v$ is the speed of the vehicle; $F_{i}$ is the ramp resistance, $F_{i}=M g \sin \alpha ; F_{j}$ is accelerated resistance.

\subsection{Driving force of maximum speed}

When the vehicle is traveling at the highest speed on the ground, The climb gradient $\alpha=\mathbf{0}$, so the ramp resistance $F_{i}=M g \sin \alpha=\mathbf{0}$, The acceleration resistance $F_{j}=\mathbf{0}$. According to the design of the maximum speed, the wheel needs the driving force:

$$
F_{t}=F_{f}+F_{w}
$$


Given the parameters of the vehicle, you can determine the drive force required for the wheel,

Where $M=\mathbf{8 4 0 0 0 \mathrm { kg }}, C_{D}=\mathbf{0 . 8}, f=\mathbf{0 . 0 1 2}, A=5.5 \mathrm{~m}^{2}, v=\mathbf{8 0} \mathrm{km} / \mathrm{h}$, the driving force required for the maximum speed $F_{t}=\mathbf{1 1 . 2} \mathrm{kN}$.

\subsection{Maximum driving force when climbing}

When the vehicle is running on the maximum slope, the speed of travel is relatively low, can ignore the wind resistance, driving resistance only rolling resistance and ramp resistance, which:

$$
F_{t}=F_{f}+F_{i}
$$

According to $30 \%$ of the gradient, $\alpha=\arctan (\mathbf{0 . 3})=16.67^{\circ}$, the driving force required for the wheel at the maximum slope is $F_{t}=24.6 \mathrm{kN}$.

\section{Parameter design of hydraulic Motor Hydraulic Motor Performance}

In the hydraulic motor selection calculation, to make the hydraulic motor to meet the hydraulic drive vehicle power requirements and the maximum speed requirements, and can zero displacement. The vehicle has the greatest running resistance at the maximum slope, and the speed of the hydraulic motor is required to meet the requirements of the running speed when traveling on the ground.

The driving torque of the hydrostatic transmission vehicle:

$$
T_{m}=\frac{F_{t} R_{r}}{n i_{g} \eta_{g}}
$$

Where $n$ is the number of drive wheels when driving, $i_{g}$ is the gear ratio of the wheel reducer, $\eta_{g}$ is the mechanical efficiency of the gear unit, $R_{r}$ is the rolling radius of the wheel.

The hydraulic motor is also required to meet the maximum speed of the vehicle design, The maximum speed of the design is $v_{\max }=\mathbf{8 0} \mathrm{km} / \mathrm{h}$. Calculate the maximum speed of the hydraulic motor according to the design maximum speed..

$$
n_{m \max }=\frac{v_{\max } 60}{2 \pi R_{r} 3.6} i_{g}
$$

Where $n_{m \max }$ is the maximum speed of the hydraulic motor, $i_{g}$ for the wheel reducer gear ratio.

\section{System work pressure calculation}

$\square$ he working pressure of the hydraulic motor is determined by the running resistance of the hydrostatic transmission vehicle. The working pressure is calculated as:

$$
\Delta P_{m}=\frac{100 T_{m}}{1.59 q_{m} \eta_{m h}}
$$

Where $\Delta P_{m}$ is the working pressure at both ends of the hydraulic motor, $T_{m}$ is the torque on the hydraulic motor, $q_{m}$ is the displacement of the hydraulic motor, $\eta_{m h}$ is the mechanical efficiency of the hydraulic motor.

\section{Parameter design of hydraulic pump}

Hydraulic pump in the calculation of parameters to meet the system's working pressure and hydraulic motor hydraulic flow requirements. Hydrostatic transmission vehicles in the ground to 
high speed, the hydraulic motor displacement to take $50 \mathrm{ml} / \mathrm{r}$, according to the number of drive the number of systems required flow:

$$
Q_{\max }=n_{\max } q_{m} n
$$

Where $n_{m \max }$ is the maximum speed of the hydraulic motor, $q_{m}$ is the displacement of the hydraulic motor, $n$ is the number of drive wheels.

Calculate the displacement of the hydraulic pump based on the maximum flow required by the system and the speed of the engine.

$$
q_{b}=\frac{Q_{\max }}{n_{b} n_{p}}
$$

Where $n_{b}$ is the number of hydraulic pumps, $n_{p}$ is the maximum speed at which the engine is operating.

\section{Engineering case analysis}

According to the vehicle driving conditions, multi-axis hydrostatic transmission vehicle driving system hydraulic pump selection Rexroth A11VO190 variable pump, hydraulic motor selection A6VM200 variable motor, A11VO190 variable pump nominal pressure of 350bar, A6VM200 variable motor nominal pressure of 400bar。According to (6) and (8) to calculate the hydraulic motor working pressure range of 174bar - 318bar, can make the system pressure to get a better match.

\section{Conclusion}

In this paper, the principle of multi-axis hydrostatic transmission vehicle is analyzed, and the parameters of the driving system, the running resistance, the hydraulic motor and the hydraulic pump are calculated and analyzed. The calculation of the parameters of the hydraulic drive system is illustrated by an example. , Which provides the basis for the calculation of the parameters of hydraulic transmission vehicles.

\section{Acknowledgment}

The article was supported by Special research project of Shaanxi Provincial Department of Education (Grant No. 14JK1134), Natural Science Project of Shaanxi Provincial Science and Technology Department (Grant No. 2016JM5039), Shaanxi Province Natural Science Basic Research Project (Grant No. 2017JM5087).

\section{References}

[1] Yao Huaixin. "Construction machinery chassis and its hydraulic drive theory”, China Communications Press,2002

[2] Chen Jinquan, Hu Junke, Zhang Zhengming. “Application of Hydrostatic Driving System in High - speed Off - road Forklift”. Modern manufacturing engineering, 2010(4),pp123-127.

[3] Wang Xin, Zhang Chao, Yi Xiaogang.” Research on Power - Load Adaptive Method for Full Hydraulic Leveling Machine”, 2007,(38),pp27-30.

[4] Geng Lingxin, Zhang Lijuan. "Matching and Simulation Analysis of Hydrostatic Transmission System for Grader”, Hydraulic and pneumatic, 2013(8),pp86-88

[5] Zuo Delong, Yin Wenqing, Hu Fei . "Matching and Simulation of Engine and Hydrostatic Transmission”, Mechanical transmission, 2010,(34),pp64-67. 
[6] ZHAO Liang. "Matching and control study on driving system of full hydraulically-driven chassis”,Journal of China Construction Machinery Corporation,2013,(6),pp490-493

[7] ZHAO Liang, DING Li-lei, TIAN Wen-peng etc.” Research on simulation for starting pressure of hydraulic transmission vehicle”, Modern Manufacturing Engineering,2014(5),pp127-130

[8] Yi Xiaogang, Jiao Shengjie, Liu Zhengfu.” Research on Key Technical Parameters of Full Hydraulic Bulldozer” China Journal of Highway and Transport,2004(2),pp119-123

[9] Mohamed Saber Ahmed Ibrahim-Sokar.Verlag.Investigation of Hydraulic Transmissions for Passenger Cars. Diss. RWTH Aachen University,2011

[10] YI Xiaogang, WANG Xin. “Matching and control techniques for hydrostatic grader”.Road Machinery \& Construction Mechanization, 2008, (3),pp18-21. 